\title{
NOELIA MARTÍNEZ DOALLO: EL DERECHO AL CONSENTIMIENTO INFORMADO A PARTIR DE LA TEORÍA DEL ESTATUS DE GEORG JELLINEK
}

\section{THE RIGHT TO INFORMED CONSENT FROM GEORG JELLINEK’ STATUS THEORY}

RESUMEN: Jellinek define "estatus" como la "relación con el Estado que califica al individuo". Su teoría distingue cuatro tipos: pasivo o subiectionis, negativo o libertatis, positivo o civitatis y activo o de la ciudadanía activa. Al margen de las polémicas sobre su vigencia, se pretende relacionar la aportación de Jellinek con la concepción del consentimiento informado del Tribunal Constitucional español, quien lo ha definido como deber de abstención de los profesionales sanitarios (STC 37/2011, de 28 de marzo, entre otras), es decir, como una negación de su competencia en la terminología de Hohfeld, o como un derecho de defensa.

Debido a la adecuación de su estructura al contenido del consentimiento informado, el análisis se centrará en los estatus negativo y positivo. La cuestión versa sobre la posición de los derechos de defensa en la tesis de Jellinek y si es viable su empleo en la conceptualización de los actuales derechos fundamentales.

ABSTRACT: Jellinek has defined "status" as the relationship between the State and the individual that qualifies to the last one. His theory distinguishes four types: passive or subiectionis, negative or libertatis, positive or civitatis and active or status of active citizenship. Besides controversies about its validity, it is aimed here to relate Jellinek's contribution to the conception of informed consent developed by the Spanish Constitutional Court, as a duty to refrain for healthcare professionals (STC 37/2001, among others), i.e., a denial of their power in Hohfeldian words, or a defence right.

\footnotetext{
* Universidade da Coruña. Contratada predoctoral FPI. Departamento de Derecho Público Especial. Grupo de investigación Filosofía, Constitución y Racionalidad. Campus de Elviña s/n, 15071 A Coruña (España) noelia.doallo@udc.es El presente artículo es un trabajo original e inédito y se configura como la versión escrita de la comunicación presentada bajo el mismo título en el Congreso Internacional: Ciencia Biomédica y Derechos Fundamentales: Nuevos Retos Compartidos, organizado por el grupo de investigación SEJ-504 Bioderecho Internacional, Red de Excelencia RIECOMM (DER2015-71828-REDT) del Ministerio de Economía y Competitividad de España, los días 1 y 2 de diciembre de 2016 en la Facultad de Derecho de la Universidad de Sevilla. Asimismo, este artículo forma parte de los resultados del proyecto de investigación "El discurso de los bioderechos. Bases filosóficas y jurídicas para su fundamentación, caracterización y aplicación" (DER2014-52811-P), financiado por el Ministerio de Economía y Competitividad.
} 
DOI: http://dx.doi.org/10.12795/IETSCIENTIA.2017.i01.19

This analysis focuses on negative and positive statuses, since both are suitable for the structure of informed consent. The issue is about the position of defence rights within Jellinek's theory and whether it is possible to use it to conceptualize contemporary fundamental rights.

PALABRAS CLAVE: Alexy, consentimiento informado, derecho al consentimiento informado, derecho de defensa, derechos fundamentales, derechos públicos subjetivos, derecho subjetivo, estatus, Hohfeld, Jellinek.

KEYWORDS: Alexy, defence right, fundamental rights, Hohfeld, informed consent, Jellinek, right to informed consent, status, subjective public rights, subjective right.

\section{INTRODUCCIÓN}

Al margen de las discusiones doctrinales en torno a la vigencia de la teoría del estatus de Jellinek, se pretende aquí "rescatar" la noción de estatus y su clasificación con el objetivo de ponerla en relación con un derecho fundamental concreto y actual cual es el derecho al consentimiento informado del paciente o usuario. Con ello se pretende comprobar si realmente es posible el acomodo de una norma jurídica de derecho fundamental como la propuesta a la estructura del estatus de Jellinek, con el objeto, más allá de verificar una pretendida vigencia, de encontrar cimientos sólidos para la conceptualización de los derechos fundamentales en general, y del derecho al consentimiento informado en particular. Así mismo, se tendrán presentes algunos de los ajustes propuestos a la tesis de Jellinek por parte de la doctrina, con especial atención a la aportación del profesor alemán Robert Alexy. Además, se ha de destacar que el análisis versará sobre la norma de derecho fundamental que nos ocupa, esto es, el derecho al consentimiento informado en tanto que deber de abstención de los profesionales sanitarios, negación de la competencia de los mismos y, en definitiva, derecho de defensa, como así lo ha venido afirmando nuestro Tribunal Constitucional en su jurisprudencia ${ }^{1}$.

Para lograr los objetivos descritos se ha creído oportuno centrar la atención en dos de los estatus propuestos por Jellinek, cuales son el estatus negativo o libertatis y el estatus positivo o civitatis. Ambos se presentan adecuados para la consideración del derecho al consentimiento informado, dado que las pretensiones derivadas del mismo concuerdan con las estructuras descritas. Cabe destacar, a modo de hipótesis, que su encaje en dos de los estatus o situaciones es significativo y avanza la constatación del carácter

\footnotetext{
${ }^{1}$ STC 37/2011, de 28 de marzo.
} 
DOI: http://dx.doi.org/10.12795/IETSCIENTIA.2017.i01.19

vacilante de la aportación de Jellinek, por lo que se anticipa la necesidad de efectuar determinados ajustes.

En primer lugar se describirá la teoría del estatus de Jellinek, así como su repercusión doctrinal, donde brevemente se dará cuenta de algunas polémicas acerca de su vigencia. En un segundo estadio, resulta imprescindible estudiar el fundamento del derecho al consentimiento informado tal y como ha sido concebido por nuestra jurisprudencia constitucional, para posteriormente poder someterlo a la estructura trazada por Jellinek. Ello permitirá obtener conclusiones acerca de la utilidad de la teoría del estatus de Jellinek en la tarea de fundamentación de los derechos fundamentales, y particularmente del derecho al consentimiento informado.

\section{LA TEORÍA DEL ESTATUS DE JELLINEK}

La teoría de Georg Jellinek enraíza con una tendencia en la ciencia jurídica alemana de finales del siglo XIX y principios del XX que se muestra crítica con las concepciones iusnaturalistas de los derechos humanos. El triunfo de la mentalidad positivista trató de ofrecer una configuración positiva de las libertades individuales frente al Estado, a estos efectos se le dotó de personalidad jurídica con el objeto de hacerlo titular de derechos y obligaciones para con los súbditos, junto con el establecimiento de garantías jurisdiccionales para la protección de estas nuevas realidades subjetivas. En este contexto nace la noción de derechos públicos subjetivos, de la cual Jellinek es uno de los principales representantes ${ }^{2}$. El surgimiento de esta nueva categoría pretendió asentar los derechos humanos en el seno de un sistema de relaciones jurídicas entre el Estado y los particulares, lo cual constituyó una tentativa de conciliar la teoría de los derechos humanos con las tesis de corte positivista. Inserto en esta tendencia, Jellinek desarrolla una "teoría de posiciones totales abstractas" 3 en la que distingue cuatro estatus diferenciados: estatus pasivo o subiectionis, estatus negativo o libertatis, estatus positivo o civitatis y estatus activo o de la ciudadanía activa ${ }^{4}$. Jellinek define el estatus

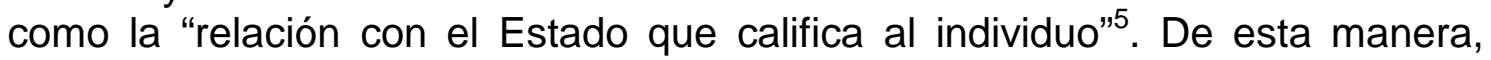
concibe al estatus como una situación y lo diferencia de un derecho, ya que el estatus tiene como contenido el "ser" jurídico y no el "tener" jurídico de una

\footnotetext{
${ }^{2}$ Cabría asimismo destacar a autores como Gerber, Laband y Mayer, quienes también tomaron parte en la construcción de dicha noción y como así lo pone de manifiesto PÉREZ LUÑO, A. E. en su obra Derechos humanos, Estado de Derecho y Constitución (1984), 10ª edición, Madrid, Tecnos, 2010, p. 60.

${ }^{3}$ ALEXY, R., Teoría de los Derechos Fundamentales (1985), traducción y estudio introductorio de Carlos Bernal Pulido, 2a edición, Madrid, Centro de Estudios Políticos y Constitucionales, 2012, pp. 219.

${ }^{4}$ JELLINEK, G., System der Subjektiven öffentlichen Rechte, 2ª edición,Tubinga, 1905, pp. 86 y ss. Vid. Alexy, R., Teoría de los..., cit., pp. 219 y ss.

${ }^{5}$ JELLINEK, G., System der Subjektiven..., cit., p. 83.
} 
DOI: http://dx.doi.org/10.12795/IETSCIENTIA.2017.i01.19

persona ${ }^{6}$. Por tanto, de estas situaciones o estatus surgen una serie de pretensiones de los ciudadanos, las cuales constituyen los denominados derechos públicos subjetivos, configurados en los cuatro estatus anteriormente enunciados.

En primer lugar, el estatus pasivo o subiectionis se configura como la abstracción de determinadas posiciones descriptibles a partir de las modalidades normativas del mandato, la prohibición, la competencia y la sujeción, y reducible a las mismas. Para que un individuo se sitúe en este estatus pasivo, éste ha de encontrarse dentro de la esfera del deber individual, en virtud de una relación de sumisión respecto del Estado ${ }^{7}$.

En segundo puesto, Jellinek configura el estatus negativo o libertatis como una esfera de libertad individual ${ }^{8}$. Bajo este estatus el individuo se sitúa en el terreno de la autonomía, dentro del cual sus actos quedan libres de la injerencia del poder estatal, por lo que el estatus negativo conlleva una garantía frente a la intromisión del Estado en determinados ámbitos ${ }^{9}$.

El tercer lugar lo ocupa el estatus positivo o civitatis. Este estatus se muestra para el propio Jellinek como un reflejo del estatus negativo, en tanto que se configura como el derecho del ciudadano frente al Estado a la realización de acciones por parte de este último ${ }^{10}$. De este modo, se encuentra en un estatus positivo aquel individuo a quien el Estado reconoce capacidad jurídica para reclamar el poder estatal para sí, con el objeto de usar las instituciones estatales ${ }^{11}$.

En cuarta y última posición se encuentra el estatus activo o de la ciudadanía activa. En este caso, su estructura formal queda suficientemente caracterizada a través del concepto de competencia. Se trata aquí de la atribución al individuo de competencias que tienen como objeto su participación

\footnotetext{
${ }^{6}$ JELLINEK, G., System der Subjektiven..., cit., p. 84.

7 JELLINEK, G., System der Subjektiven..., cit., p. 86.

${ }^{8}$ Jellinek entiende la expresión "esfera de libertad individual" como la clase "de las acciones de los súbditos jurídicamente irrelevantes para el Estado". Vid. JELLINEK, G., System der Subjektiven..., cit., p. 104.

${ }^{9}$ Este estatus resulta más controvertido, puesto que no queda claro si se trata de simples posiciones libres, esto es, libertades jurídicas no protegidas; o, por el contrario, también se incluyen protecciones a dichas libertades en la forma de derechos a la no obstaculización, esta cuestión será tratada más abajo en relación a la posible inclusión o no de los derechos de defensa en este estatus, lo cual dependerá en gran medida de la definición de "irrelevancia jurídica".

${ }_{10}$ JELLINEK, G., System der Subjektiven..., cit., p. 121.

${ }^{11}$ Vid. JELLINEK, G., System der Subjektiven..., cit., p. 87. Posteriormente se volverá sobre el estatus positivo o civitatis para tratar de dilucidar si es en sede del estatus positivo donde se han de insertar los derechos de defensa, o si tan solo puede integrar su contenido "un hacer positivo del Estado". Vid. JELLINEK, G., System der Subjektiven..., cit., p. 121.
} 
DOI: http://dx.doi.org/10.12795/IETSCIENTIA.2017.i01.19

en el Estado, y encaminadas a la creación de la voluntad estatal ${ }^{12}$. Con todo, no resulta clara su delimitación respecto del estatus positivo, pues existen casos dudosos en los que estatus activo y positivo aparecen vinculados, como por ejemplo en el supuesto del derecho al voto. Estas oscilaciones son representativas de un carácter relativamente difuso presente, en mayor o menor medida, en toda la teoría de Jellinek ${ }^{13}$.

\section{CONSIDERACIONES ACERCA DE LA TEORÍA DEL ESTATUS DE JELLINEK}

Existen diferentes posturas al respecto de la vigencia de la teoría del estatus de Jellinek. Un sector de la doctrina destaca la necesidad de su consideración no solo en virtud de su gran conceptualización y teorización jurídicas, sino también debido a su vigencia actual como base para la clasificación de los derechos fundamentales ${ }^{14}$. Dicha validez vendría abalada por los intentos de acomodar la estructura de la tesis de Jellinek, ciertamente influenciada por la mentalidad del Estado liberal de Derecho, al modelo del Estado social de Derecho ${ }^{15}$. No obstante, otros autores discrepan de su vigencia, y emplean justo en sentido contrario estas tentativas de acomodar la teoría del estatus a las nuevas realidades. En este sentido, Pérez Luño opina que "[l]a propia sutileza de estos esfuerzos teóricos por acomodar la noción clásica de derechos públicos subjetivos a las nuevas situaciones surgidas de las nuevas circunstancias no supone sino tentativas de injertar nueva savia en un tronco caduco"16, además de suponer una distorsión de las estructuras de Jellinek, con las cuales resultan incompatibles. Este autor concibe la tesis de Jellinek como una concepción de marcado corte liberal burgués que de ninguna manera resulta trasladable a los nuevos modelos derivados del avance económico y social, y por lo tanto obsoleta. Representativo de ello resulta el progresivo abandono de la noción de derechos públicos subjetivos en favor de una noción más amplia de los derechos fundamentales ${ }^{17}$.

\footnotetext{
12 JELLINEK, G., System der Subjektiven..., cit., p. 136 y 137.

${ }^{13}$ ALEXY, R., Teoría de los..., cit., p. 232.

${ }^{14}$ Son de esta opinión: Fr. Klein, W. Schmitt Glaeser, J. Schwabe o C. Starck, así como el propio R. Alexy. Vid. ALEXY, R., Teoría de los..., cit., p. 219.

${ }^{15}$ Ejemplos de ello son los esfuerzos de P. Häberle de adaptar la teoría de Jellinek a la mentalidad jurídica democrática, quien intentó complementar el elenco de estatus de Jellinek con su estatus activus processualis. En este mismo sentido, destacan las propuestas de E. Denninger y su estatus constituens; $\mathrm{K}$. Hesse con sus estatus de derechos constitucional y derecho político general; o Grabitz y su estatus libertatis. Vid. ALEXY, R., Teoría de los..., cit., pp. 219 y 220. En España destaca E. García de Enterría, quien propuso en el ámbito del derecho administrativo la distinción de dos tipos de derechos públicos subjetivos: típicos o activos y reaccionales o impugnatorios. Vid. PÉREZ LUÑO, A. E., Derechos humanos, Estado..., cit., p. 35.
}

${ }_{17}^{16}$ PÉREZ LUÑO, A. E., Derechos humanos, Estado..., cit., p. 36.

${ }^{17}$ PÉREZ LUÑO, A. E., Derechos humanos, Estado..., cit., p. 35. 


\section{EL CONSENTIMIENTO INFORMADO EN LA JURISPRUDENCIA CONSTITUCIONAL ESPAÑOLA}

Resulta vital para el objeto de estudio propuesto tener constancia de la fundamentación efectuada por nuestra jurisprudencia constitucional acerca del derecho al consentimiento informado antes de ponerlo en relación con la teoría de Jellinek. Así pues, el consentimiento informado pertenece al ámbito de la autonomía decisoria del paciente, esto es, se configura como una libertad de elección bajo tres requisitos, cuales son la información, la voluntariedad y la capacidad $^{18}$. Así lo ha reconocido nuestro Tribunal Constitucional en múltiples ocasiones al afirmar que el consentimiento informado es una facultad de autodeterminación que legitima al paciente para decidir libremente sobre las medidas terapéuticas y tratamientos que puedan afectar a su integridad, a pesar de que pueda conducir a un resultado fatal ${ }^{19}$. Sin embargo, el propio Tribunal Constitucional ha señalado reiteradamente que el consentimiento informado no tiene cabida en el ámbito de la libertad personal o física, alegando que el artículo 17 de la Constitución española no recoge una libertad general de autodeterminación individual ${ }^{20}$. La libertad constituye un valor superior de nuestro ordenamiento jurídico, como así lo establece el artículo 1.1 de la Constitución española al estipular como valores superiores del ordenamiento jurídico la libertad, la justicia, la igualdad y el pluralismo político, mas solo procederá su protección mediante recurso de amparo en presencia de un derecho fundamental; en otras palabras, el recurso de amparo solo protegerá las eventuales vulneraciones de los derechos explícitamente recogidos en los artículos 14 a 29 y 30.2 de la Constitución española. Esto supone que nuestro texto constitucional no recoge expresamente un derecho a la autonomía, ni un derecho general de libertad $^{21}$, no existiendo un reconocimiento expreso del derecho al consentimiento informado en dicho texto.

No obstante, la jurisprudencia constitucional ha atribuido el consentimiento informado al artículo $15 \mathrm{CE}$, el cual "protege la inviolabilidad de la persona, no solo contra ataques dirigidos a lesionar su cuerpo y espíritu, sino también contra toda clase de intervención en esos bienes que carezca del

\footnotetext{
${ }^{18}$ SEOANE, J. A., "La construcción jurídica de la autonomía del paciente", EIDON 39, 2013, p. 30.

${ }_{19}$ Ilustran esta concepción del consentimiento informado las Sentencias del Tribunal Constitucional 120/1990, de 27 de junio, FJ 8ㅇ; 154/2002, de 18 de julio, FJ 9o; 37/2011, de 28 de marzo, FJ 5으, entre otras.

${ }^{20}$ Vid. SSTC126/1987, de 16 de julio; 22/1988, de 18 de febrero; 12/1988, de 3 de febrero; $61 / 1990$, de 29 de marzo, y más recientemente la 37/2011, de 28 de marzo, FJ $3^{\circ}$.

${ }^{21}$ Vid. a contrario PRIETO SANCHÍS, L., Estudios sobre derechos fundamentales, Madrid, Debate, 1990, p. 157 y ss.
} 
consentimiento de su titular"22, por lo que dicha adscripción posibilita la clasificación del derecho al consentimiento informado como una norma de derecho fundamental ${ }^{23}$. De este modo, resulta posible afirmar que nuestro Tribunal Constitucional configura el consentimiento informado como una facultad negativa, es decir, un deber de abstención del médico con un sustrato corporal. La tesis descrita concuerda ampliamente con los "conceptos jurídicos fundamentales" del profesor norteamericano W. N. Hohfeld, pues el paciente ostenta una inmunidad que le permite rechazar una intervención en contra de su voluntad, lo cual es correlato de la incompetencia del médico ${ }^{24}$, quien no podrá iniciar las actuaciones médicas sin haber obtenido previo consentimiento del paciente. Como se podrá observar, el encaje conceptual es idóneo.

Entender el derecho al consentimiento informado como un mero derecho de defensa puede ser discutido, mas este no es el objeto de análisis aquí, por lo que se seguirá la línea marcada por nuestra Jurisprudencia Constitucional, en tanto que material autoritativo, sin más consideraciones. La intención principal es concluir sí, desde el punto de vista teórico y analítico, resulta posible reconducir el paradigma de Jellinek al actual modelo de los derechos fundamentales, en aras de alcanzar mayor claridad al respecto de su conceptualización y clasificación.

\section{EL DERECHO AL CONSENTIMIENTO INFORMADO Y EL ESTATUS NEGATIVO O ESTATUS LIBERTATIS}

A pesar de que las consideraciones del propio Jellinek hacen descartar la posibilidad de incluir a los denominados derechos de defensa en el marco del estatus negativo, si resultaría posible tal circunstancia mediante la realización de ciertas matizaciones. Hacía este objetivo caminan las consideraciones de Alexy al analizar el mencionado estatus negativo ${ }^{25}$.

La imposibilidad original de incluir a los derechos de defensa, y por tanto al derecho al consentimiento informado, en la categoría del estatus negativo proviene de la afirmación del propio Jellinek acerca de que dicho estatus se trata de una "esfera de libertad individual", la cual viene determinada por la clase "de las acciones de los súbditos jurídicamente irrelevantes para el Estado" 26 . A su vez, en la tesis de Jellinek se ha de entender "acción jurídicamente irrelevante" como aquella que no puede provocar un efecto

\footnotetext{
${ }^{22}$ Vid. SSTC 120/90, de 27 de junio, FJ 8º; y 119/2001, de 24 de mayo, FJ 5o.

${ }^{23} \mathrm{Al}$ respecto del concepto de "norma adscrita de derecho fundamental", vid. ALEXY, R., Teoría de los..., cit., p. 52.

${ }^{24} \mathrm{Al}$ respecto de los conceptos de inmunidad y competencia, vid. HOHFELD, W. N., "Some Fundamental Legal Conceptions as applied in Judicial Reasoning I", Yale Law Journal, XXIII, n. I, 1913, p. 39.

${ }^{25}$ ALEXY, R., Teoría de los..., cit., p. 222.

${ }^{26}$ JELLINEK, G., System der Subjektiven..., cit., p. 104.
} 
jurídicamente relevante ${ }^{27}$. Dicha irrelevancia se remite a las posiciones libres, esto es, aquellas que ni están ordenadas ni prohibidas, de modo que se tratan de libertades jurídicas no protegidas. En este punto surgen las objeciones derivadas de la inclusión de los derechos de defensa en esta estructura, en tanto que dichos derechos de defensa suponen algún tipo de protección o garantía, por lo que no resultarían jurídicamente irrelevantes. Esta consideración imposibilitaría la inserción de los derechos de defensa en el status negativo, corriéndose el riesgo de que esta importante categoría quedara excluida de la estructura propuesta por Jellinek ${ }^{28}$, lo cual desacreditaría considerablemente su utilidad.

De todos modos, el propio Jellinek ha afirmado que "el estatus negativo está protegido porque el individuo tiene pretensión a su reconocimiento y a las autoridades estatales les está prohibida toda perturbación del mismo"29. Esta afirmación, además de antojarse contradictoria con la anterior apreciación acerca de las libertades jurídicas no protegidas, indica un cierto tipo de protección, de modo que cabe matizar la irrelevancia de las acciones recogidas por el estatus negativo. Se podría decir que estamos ante uno de los ejemplos del carácter oscilante de la tesis del estatus de Jellinek.

Con la intención de salvar este tipo de contradicciones, Alexy ha propuesto la subdivisión del estatus negativo en dos "subestatus". De este modo, obtiene un "estatus negativo en sentido estricto", exclusivamente referido a las libertades no protegidas; y un "estatus negativo en sentido amplio", el cual también incluye los derechos a acciones negativas del Estado, es decir, los derechos de defensa ${ }^{30}$. Una vez realizadas estas consideraciones e introducidas en la teoría del estatus, resultaría posible incluir al derecho al consentimiento informado, en tanto que derecho de defensa, en el seno del "estatus negativo en sentido amplio".

\section{EL DERECHO AL CONSENTIMIENTO INFORMADO Y EL ESTATUS POSITIVO O CIVITATIS}

Como ya se ha dicho, el estatus positivo resulta ser reflejo del estatus negativo, en tanto que derecho del ciudadano a acciones estatales frente al Estado. De esta manera, el contenido del estatus positivo aparece circunscrito al establecimiento o no de la libertad del Estado para ejecutar u omitir una acción ${ }^{31}$. Jellinek incluye en el estatus positivo del individuo la pretensión frente

\footnotetext{
${ }^{27}$ JELLINEK, G., System der Subjektiven..., cit., p. 46. Se ha de destacar en este punto, a modo de crítica, que Jellinek efectúa una definición tautológica y poco apta para el esclarecimiento de su concepto de "relevancia jurídica".

${ }^{28}$ ALEXY, R., Teoría de los..., cit., p. 230.

${ }^{29}$ JELLINEK, G., System der Subjektiven..., cit., p. 105.

${ }^{30}$ ALEXY, R., Teoría de los..., cit., p. 230.

${ }^{31}$ JELLINEK, G., System der Subjektiven..., cit., p. 121.
} 
a las autoridades estatales de no impedimento ${ }^{32}$, como "toda pretensión a un determinado comportamiento estatal, ${ }^{33}$, lo cual supone un derecho frente al Estado respecto de una acción negativa, es decir, una omisión ${ }^{34}$.

Sin embargo, la adscripción de la omisión de impedimentos al estatus positivo se opone a la tesis de Jellinek, al definir el contenido de los derechos que han de vincularse al estatus positivo como aquellos consistentes en "un hacer positivo del Estado"35, de manera que una omisión de impedimentos nunca podría incluirse en dicha categoría, ya que, aun existiendo la posibilidad de definirlo como "un hacer", en ningún caso se podría categorizar como "un hacer positivo" 36 .

Por todo lo anterior, los derechos de defensa quedarían fuera de la estructura propuesta por Jellinek. Para evitar que esto ocurra Alexy plantea de nuevo la posibilidad de insertar los derechos de defensa en el estatus positivo mediante la subdivisión del estatus positivo en dos, de forma que obtiene un "estatus positivo en sentido estricto", y "un estatus positivo en sentido amplio". De este modo, pertenecerían al "estatus positivo en sentido amplio" tanto los derechos a acciones positivas como los derechos a acciones negativas del Estado, es decir, los derechos de defensa. En cambio, en el "estatus positivo en sentido estricto" tan solo resultaría posible incluir aquellos derechos a acciones positivas del Estado ${ }^{37}$.

De lo anterior cabe concluir que los derechos de defensa se suscriben al estatus positivo "en sentido amplio", por lo que el derecho al consentimiento informado, en tanto que derecho de defensa, se integrará aquí. Sin embargo, si tomamos como referencia el derecho al consentimiento informado en sí mismo considerado, podemos preguntarnos acerca de su contenido prestacional. Si la respuesta es afirmativa, y el derecho al consentimiento informado goza de contenido prestacional, también resultaría pertinente incluirlo en el estatus positivo "en sentido estricto". El contenido prestacional del consentimiento informado vendría justificado por el deber inexcusable, salvo en los supuestos legalmente previstos, de informar al paciente, lo cual supone la puesta a disposición de medios, y, por tanto, "un hacer positivo". De todos modos, lo anteriormente expuesto nos dirige hacia la discusión acerca del fundamento del consentimiento informado, la cual no se pretende abordar aquí. Si bien, el esquema de Jellinek nos puede poner en la pista de algún desajuste en la consideración consentimiento informado como mero derecho de defensa, dado que no tendría en cuenta su naturaleza prestacional.

\section{CONCLUSIÓN}

\footnotetext{
${ }^{32}$ JELLINEK, G., System der Subjektiven..., cit., p. 111.

${ }^{33}$ JELLINEK, G., System der Subjektiven..., cit., p. 105.

${ }^{34}$ ALEXY, R., Teoría de los..., cit., p. 229.

35 JELLINEK, G., System der Subjektiven..., cit., p. 121.

${ }^{36}$ ALEXY, R., Teoría de los..., cit., p. 230.

${ }^{37}$ ALEXY, R., Teoría de los..., cit., p. 230.
} 
Como se ha tenido oportunidad de observar, la teoría del estatus de Jellinek presenta algunas carencias al respecto de la inclusión de los derechos de defensa en su estructura, categoría a la cual la jurisprudencia constitucional ha adscrito el derecho al consentimiento informado del paciente o usuario. Por una parte, presenta un carácter oscilante y contradictorio al hacerlo pivotar entre los estatus negativo y positivo; por otra, se observan algunas ambigüedades conceptuales que necesitan ser matizadas. A pesar de las dificultades destacadas, resulta esclarecedor emplear la tesis de Jellinek para analizar normas de derecho fundamental como la propuesta, por lo que se ha tenido a bien proponerla como una teoría a considerar en el análisis de la estructura de los bioderechos.

Además se podría pensar que de la propia definición de estatus efectuada por Jellinek, como la relación entre Estado e individuo que caracteriza a este último, resulta un escollo quizá insalvable para la trasposición de su tesis al actual modelo de los derechos fundamentales. Sin embargo, se ha de tener presente que en Jellinek estatus y derecho son realidades distintas, siendo el estatus una situación previa en la que los individuos se sitúan y en virtud de la cual se adscriben los derechos, en tanto que pretensiones institucionalmente reconocidas y garantizadas por los poderes públicos, lo cual no difiere respecto del actual modelo de protección de los derechos fundamentales.

Se ha tenido ocasión de comprobar la adecuación de incluir el derecho al consentimiento informado dentro del estatus negativo, en tanto que es posible afirmar que el sometimiento a un tratamiento o terapia no está ni ordenado ni prohibido, estando permitida tanto su ejecución como su omisión, por lo que se encuentra dentro del ámbito de libertad del paciente, lo cual encaja con el concepto de derecho de defensa sin mayores inconvenientes. Por lo que respecta al estatus positivo, también resulta congruente incluir aquí al derecho al consentimiento informado en tanto que contiene una parte positiva, cual es la recepción por parte del paciente o usuario de la información relevante para su salud, sin embargo ello nos alejaría de la conceptualización del derecho al consentimiento informado entendido como derecho de defensa efectuada por nuestra jurisprudencia constitucional y nos llevaría a replantearnos el fundamento constitucional del mencionado derecho. No obstante, también la libre decisión y eventual negativa del paciente o usuario a someterse a un tratamiento o terapia tiene cabida en sede del estatus positivo, puesto que, entendido éste "en sentido amplio", también podría incluir un deber de omisión de una acción por parte del Estado.

\section{BIBLIOGRAFÍA CITADA}


ALEXY, R., Teoría de los Derechos Fundamentales (1985), traducción y estudio introductorio de Carlos Bernal Pulido, $2^{\text {a }}$ edición, Madrid, Centro de Estudios Políticos y Constitucionales, 2012

HOHFELD, W. N., "Some Fundamental Legal Conceptions as applied in Judicial Reasoning I", Yale Law Journal, XXIII, n. I, 1913

JELLINEK, G., System der Subjektiven öffentlichen Rechte, $2^{\mathrm{a}}$ edición,Tubinga, 1905

PÉREZ LUÑO, A. E., Derechos humanos, Estado de Derecho y Constitución (1984), 10ª edición, Madrid, Tecnos, 2010

PRIETO SANCHÍS, L., Estudios sobre derechos fundamentales, Madrid, Debate, 1990

SEOANE, J. A., "La construcción jurídica de la autonomía del paciente", EIDON 39,2013

\section{REFERENCIAS NORMATIVAS}

STC 37/2011, de 28 de marzo

STC 154/2002, de 18 de julio

STC 119/2001, de 24 de mayo

STC 120/1990, de 27 de junio

STC $61 / 1990$, de 29 de marzo

STC 22/1988, de 18 de febrero

STC $12 / 1988$, de 3 de febrero

STC 126/1987, de 16 de julio 